\title{
EDITORIAL
}

\section{THE COMMUNICATION BARRIER: DRUG DISCOVERY'S FINAL FRONTIER?}

\author{
Enthusiasm for adopting new approaches to drug discovery seems to stop short of how \\ researchers communicate their ideas. The time has come to reinvigorate public forums of debate.
}

Despite ongoing efforts on the part of the pharmaceutical industry to bring their research scientists into closer contact with their colleagues in universities, the two cultures — industry and academia — are still worlds apart. In an attempt to narrow the gap, this month's edition of Nature Reviews Drug Discovery - unusual in that it devotes almost half the issue to a single topic - brings the views of 20 leading academic authorities on ion channels to bear on some of the key questions facing industrial researchers. The state of ion channel research in 2004 (page 237) highlights both the underlying complexity of the ion channel field, and the attendant problems of attempting to therapeutically target ion channels, as well as offering a fresh perspective on areas of promise. As explained on page 238, the format is an experimental one, designed to bring many differing points of view to our attention. Encountering such diversity of opinion within a constrained space is surprising and, we hope, thought provoking.

Experimenting with 'Symposia in print', such as the new Twenty Questions section, is one way to expand the journal's coverage of a range of ideas and promote cross-fertilization between disciplines and research environments. That such cross-fertilization is badly needed and will benefit drug discovery efforts is commonly acknowledged; however, rather surprisingly for a field frequently characterized by its limitless enthusiasm for embracing novel methodologies, the drug discovery community's approach to the communication of ideas, at least outside company walls, is often extraordinarily conservative and reserved. Drug discovery conferences, which the uninitiated might surely identify as the most promising venue for the potential marriage of minds, generally tend to feature lacklustre talks with little or no ensuing discussion. Of course, concerns about intellectual property constrain what can be said, but that's no excuse for the listless nature of the scientific sessions at the average meeting. What's gone wrong?
An answer frequently given is that it doesn't matter what happens within the conference halls, because the real discussion goes on in the corridors outside. Niels Bohr recounts a story of how Ernest Rutherford, who proposed the existence of the atomic nucleus, and Max Born, one of the fathers of quantum theory, first met each other while escaping from the same boring lecture at a conference on Lake Como. "You couldn't stand it either, let's have a trip on the lake", said Rutherford, and so began a lifelong friendship. And, more often than not, it's such encounters, albeit mostly on a lesser scale, that one remembers after conferences. But lively debate and chance encounters in corridors shouldn't be mutually exclusive. The question remains: why don't we care about communication in public?

Things have to get pretty bad before we make a fuss. At a drug discovery meeting earlier this year, the controversial reproductive scientist Panayiotis Zavos was invited to give an after-dinner speech. Having just days before announced at a London press conference that he had implanted a cloned human embryo into a woman's womb (a procedure that later turned out to have been unsuccessful) many in the audience were shocked by his presence and left the room before he started speaking; others walked out during the talk. Questioning was heated, and when interrogation gave way to someone hurling abuse from the floor, the compère finally stepped in to end the evening. Whatever people thought of that session, they certainly cared.

Bringing life back into the conference circuit must surely be of mutual benefit. Although this should be a shared responsibility - and conference planners, speakers and audiences all need to contribute their enthusiasm - perhaps the people best placed to effect change are those chosen to moderate the sessions. We should ask more from our scientific moderators, and help them ensure that their sessions regain their standing as valuable venues for the exchange of ideas. 